\title{
BOUNDS AND MAXIMAL SOLUTIONS FOR NONLINEAR FUNCTIONAL EQUATIONS
}

\author{
BY JAGDISH CHANDRA AND B. A. FLEISHMAN ${ }^{1}$
}

Communicated by J. B. Diaz, December 19, 1967

1. Introduction. Consider the two functional equations

$$
\begin{aligned}
& L u=N u+p, \\
& L u=M u+q
\end{aligned}
$$

in a real Banach space $B$, where $L$ is a linear operator mapping a subset of $B$ into $B ; M$ and $N$ are operators (in general, nonlinear) mapping $B$ into $B ; p$ and $q$ are fixed elements in $B$; and the following inequality holds:

$$
N u+p \leqq M u+q \text { for all } u \in B .
$$

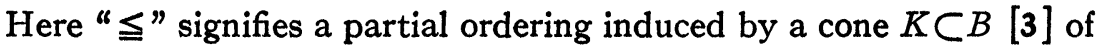
"positive" elements:

$$
u \leqq v \text { if and only if } \quad(v-u) \in K .
$$

In this paper we extend results obtained previously [2] for positive solutions (that is, solutions in $K$ ) of (1) and (2); here we consider solutions which are not necessarily in $K$. Specifically, under condition (3) and certain other assumptions, we establish below that the (unique) solution of (2) is an upper bound on all solutions of (1) (\$2); and, under additional hypotheses on $N$, we construct the "maximal" solution for (1) (\$3). Finally, we make some remarks about positive solutions ( $\$ 4)$.

Applications of these results to systems of nonlinear equations and nonlinear boundary value problems for ordinary differential equations can be found in [1], [2]. Related results in the case of (elliptic) partial differential equations have been obtained by Parter [4].

The result in $\$ 2$ might be described as a generalization of the "generalized Bellman's Lemma" (see [5]); for it follows from (3) that any solution of (1) satisfies $L u \leqq M u+q$, and integral inequalities of this form are treated in [5].

We make the following assumptions once and for all:

$\left(A_{1}\right) L$ has a bounded inverse $L^{-1}$ which is defined on $B$ and leaves the cone $K$ invariant.

1 This work has been supported by Contract AF49(638)-1661 between the office of Scientific Research of the U. S. Air Force and Rensselaer Polytechnic Institute. 
$\left(\mathrm{A}_{2}\right) \quad M$ is defined on $B$ and is Lipschitz continuous with Lipschitz constant $\beta$ satisfying

$$
\beta\left\|L^{-1}\right\|<1 \text {. }
$$

2. Upper bound. By an application of the Banach fixed point theorem, condition (4) implies that the equation (2) has a unique solution $\phi$ in $B$. We show now that all solutions of (1) (if any exist) are bounded above by $\phi$.

Theorem 1. Let $\left(\mathrm{A}_{1}\right),\left(\mathrm{A}_{2}\right)$ and (3) hold. Let either $N$ or $M$ (or both) be monotonic on $B$ :

$$
u, v \in B, \quad u \geqq v, \quad \text { imply } \quad N u \geqq N v .
$$

If $z \in B$ is a solution of (1), then

$$
z \leqq \phi \cdot
$$

Proof. Case 1. Let $M$ be monotonic. For $n=1,2, \cdots$, set

$$
\phi_{n}=L^{-1}\left(M \phi_{n-1}+q\right), \quad \phi_{0}=z .
$$

Then using (3)

$$
\phi_{0}=z=L^{-1}(N z+p) \leqq L^{-1}(M z+q)=L^{-1}\left(M \phi_{0}+q\right)=\phi_{1} .
$$

We have used here the fact that

$\{(M z+q)-(N z+p)\} \in K$, therefore $L^{-1}\{(M z+q)-(N z+p)\}$

is in $K$. Now from (5)

$$
\phi_{2}-\phi_{1}=L^{-1}\left(M \phi_{1}-M \phi_{0}\right) \in K .
$$

Thus by induction

$$
z \leqq \phi_{1} \leqq \phi_{2} \leqq \cdots \leqq \phi_{n} \leqq \cdots,
$$

that is, the sequence $\left\{\left(\phi_{n}-z\right)\right\}$ is in $K$. Since $K$ is closed $\lim \left(\phi_{n}-z\right) \in K$. But $\phi_{n}$ converges to the unique solution $\phi$ of $(2)$. Hence $(\phi-z) \in K$.

Case 2. Let $N$ be monotonic. Again $z \leqq \phi_{1}$. Now from (3) and (5) $\phi_{2}-z=L^{-1}\left[\left(M \phi_{1}+q\right)-\left(N \phi_{1}+p\right)+\left(N \phi_{1}+p\right)-(N z+p)\right] \in K$. If we assume

$$
\left(\phi_{n}-z\right) \in K,
$$

it follows similarly that

$$
\left(\phi_{n+1}-z\right) \in K
$$


Proceeding to the limit, we have $(\phi-z) \in K$. This completes the proof of Theorem 1.

An analogous result may be found for a lower bound. Specifically, if the inequality (3) is reversed and all other hypotheses in Theorem 1 remain the same, we conclude that $\phi$ (the unique solution of (2)) is a lower bound for all solutions of (1).

3. Maximal solution. We now make some additional assumptions on $N$, which will insure existence of at least one solution of (1) in $B$.

$\left(\mathrm{A}_{3}\right) N$ is defined on $B$, is completely continuous, and satisfies for all $u \in B$

$$
\|N u\| \leqq \nu\|u\|+\alpha
$$

and

$$
\nu\left\|L^{-1}\right\|<1
$$

where $\alpha$ and $\nu$ are nonnegative constants. When $\left(\mathrm{A}_{3}\right)$ holds, an application of the Schauder fixed point theorem yields the existence of at least one solution of (1) in $B$. Specifically, set

$$
u_{n}=L^{-1}\left(N u_{n-1}+p\right) \quad u_{0}=L^{-1} p .
$$

If we assume now that $\left\|u_{n}-L^{-1} p\right\| \leqq R$, it follows from (6) and (7) that $\left\|u_{n+1}-L^{-1} p\right\| \leqq R$ whenever

$$
R \geqq\left\|L^{-1}\right\|\left(\nu\left\|L^{-1} p\right\|+\alpha\right) /\left(1-\nu\left\|L^{-1}\right\|\right) .
$$

Since $N$ is completely continuous, $\left\{u_{n}\right\}$ is compact. Therefore, there exists a subsequence converging to a solution of (1).

Definition. $u \in B$ is said to be a maximal solution of (1) with respect to the ordering induced by $K$ when

(i) $u$ is a solution of (1); and

(ii) if $z$ is any other solution, then $z \leqq u$.

Theorem 2. Let $\left(\mathrm{A}_{1}\right),\left(\mathrm{A}_{2}\right),\left(\mathrm{A}_{3}\right)$ and (3) hold. Suppose $N$ is monotonic on $B$. Then there exists a maximal solution $\bar{u}$ for $(1)$, given by

$$
u=\lim u_{n}=\lim L^{-1}\left(N u_{n-1}+p\right), \quad u_{0}=\phi,
$$

where $\phi$ is the (unique) solution of (2) in $B$.

Proof. For $n=1,2, \cdots$, set

$$
u_{n}=L^{-1}\left(N u_{n-1}+p\right), \quad u_{0}=\phi .
$$

From (3)

$$
u_{0}-u_{1}=L^{-1}\left[\left(M u_{0}+q\right)-\left(N u_{0}+p\right)\right] \in K,
$$


and from the monotonicity of $N$

$$
u_{1}-u_{2}=L^{-1}\left(N u_{0}-N u_{1}\right) \in K .
$$

An induction on $n$ shows that $\left\{u_{n}\right\}$ is monotonic decreasing. If now we choose

$$
R \geqq\left\|L^{-1}\right\|\left\{(\nu\|\phi\|+\alpha)+\left\|L^{-1} p-\phi\right\|\right\} /\left(1-\nu\left\|L^{-1}\right\|\right),
$$

it is easily seen that $\left\|u_{n}-\phi\right\| \leqq R$ implies $\left\|u_{n+1}-\phi\right\| \leqq R$. Since $N$ is completely continuous, $\left\{u_{n}\right\}$ is compact. Therefore, there exists an element $\bar{u}$ in $B$ such that

$$
\bar{u}=\lim u_{n} .
$$

Here we have used the fact that a compact monotonic sequence converges [3, p. 40]. From the continuity of $N$ it follows that $\bar{u}$ is a solution of (1).

We show now that $u$ is the maximal solution. Let $z$ be any other solution of (1) in $B$. From Theorem 1

$$
z \leqq \phi=u_{0},
$$

and from (5)

$$
u_{1}-z=L^{-1}\left(N u_{0}-N z\right) \in K \text {. }
$$

By an induction on $n$, it follows that

$$
\left(u_{n}-z\right) \in K, \text { for } n=1,2, \cdots .
$$

Since $K$ is closed

$$
(\bar{u}-z) \in K \text {. }
$$

This completes the proof of Theorem 2.

In a similar manner the existence of a minimal solution of (1) may be established when inequality (3) is reversed and in the preceding proof $\phi$ is assumed to be a lower bound.

4. Positive solutions. When $M$ and $N$ leave the cone $K$ invariant, and $p$ and $q$ are in $K$, the results of $\S \S 2$ and 3 yield the corresponding results for positive solutions (that is, solutions in $K$ ) of (1) [2]. For, let the assumptions $\left(\mathrm{A}_{1}\right)$ and $\left(\mathrm{A}_{2}\right)$ hold, and let $M K \subset K$ and $q \in K$. Then it can be seen [1] that the (unique) solution $\phi$ of (2) is in $K$. Likewise, setting

$$
u_{n}=L^{-1}\left(N u_{n-1}+p\right), \quad u_{0}=L^{-1} p, \quad n=1,2, \cdots,
$$

if $\left(\mathrm{A}_{1}\right)$ and $\left(\mathrm{A}_{3}\right)$ hold, $N K \subset K$ and $p \in K$, all the iterates remain in $K$. Therefore, there exists at least one positive solution of (1). 
Now, when the inequality (3) holds, in particular, for all $u \in K$, $\phi$ will be an upper bound on all solutions of (1) in $K$. Similarly, as in Theorem 2, we can construct the maximal solution of (1) in $K$ by starting the above iteration with $\phi$.

The authors wish to express their appreciation to the members of the Seminar on Applied Functional Analysis at Rensselaer Polytechnic Institute for their helpful comments.

\section{REFERENCES}

1. J. Chandra and B. A. Fleishman, Positive solutions of nonlinear equations, Trans. 13th Conference of Army Mathematicians, 1967 (to appear).

2. - Comparison of positive solutions of nonlinear equations, Proc. U. S.Japan Seminar on Differential and Functional Equations, Benjamin, New York, 1967, pp. 435-442.

3. M. A. Krasnoselskil, Positive solutions of operator equations, Noordhoff, Groningen 1964.

4. S. V. Parter, Maximal solutions of mildly nonlinear elliptic equations, Proc. Sympos. on Numer. Solutions of Nonlinear Differential Equations, Wiley, New York, 1966, pp. 213-238.

5. B. Viswanatham, $A$ generalization of Bellman's lemma, Proc. Amer. Math. Soc. 14 (1963), 15-18.

Maggs Research Center, Watervliet, New York and Rensselaer Polytechnic Institute 